\title{
Fermi-level-dependent defect formation in Cu-chalcopyrite semiconductors
}

Cite as: Appl. Phys. Lett. 74, 2283 (1999); https://doi.org/10.1063/1.123825

Submitted: 12 January 1999 • Accepted: 20 February 1999 • Published Online: 14 April 1999

\section{A. Klein and W. Jaegermann}

\section{ARTICLES YOU MAY BE INTERESTED IN}

Effects of $\mathrm{Ga}$ addition to $\mathrm{CuInSe}_{2}$ on its electronic, structural, and defect properties

Applied Physics Letters 72, 3199 (1998); https://doi.org/10.1063/1.121548

Chalcopyrite/defect chalcopyrite heterojunctions on the basis of CulnSe 2

Journal of Applied Physics 73, 2902 (1993); https://doi.org/10.1063/1.353020

Detailed Balance Limit of Efficiency of p-n Junction Solar Cells

Journal of Applied Physics 32, 510 (1961); https://doi.org/10.1063/1.1736034

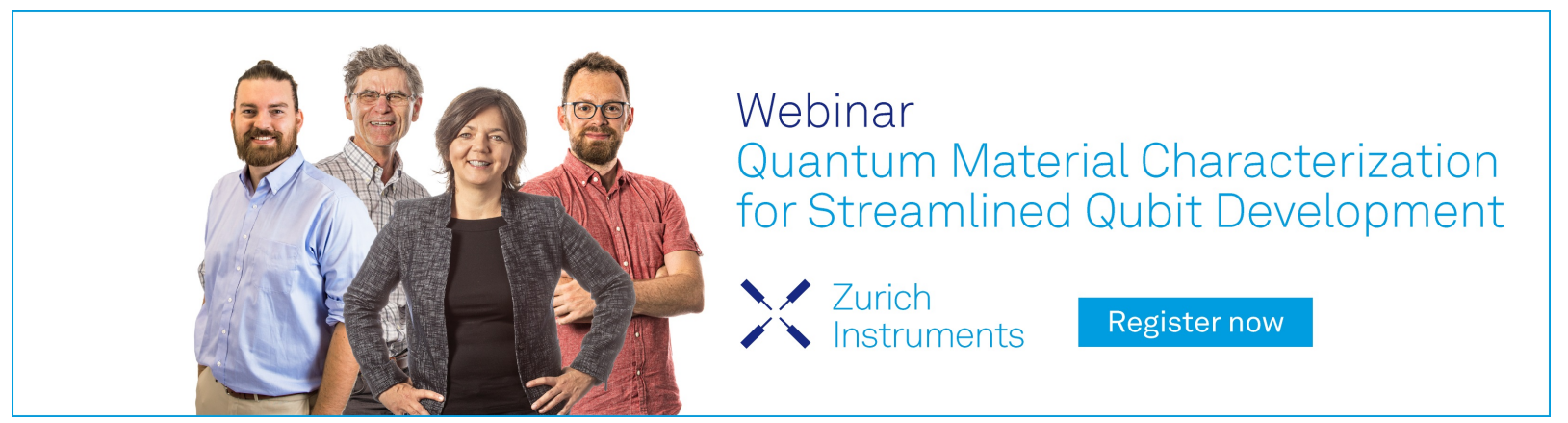




\title{
Fermi-level-dependent defect formation in Cu-chalcopyrite semiconductors
}

\author{
A. Klein ${ }^{\text {a) }}$ and W. Jaegermann \\ Department of Materials Science, Darmstadt University of Technology, Petersenstrasse 23, \\ D-64287 Darmstadt, Germany
}

(Received 12 January 1999; accepted for publication 20 February 1999)

\begin{abstract}
Valence-band photoelectron spectroscopy of $\mathrm{CuInSe}_{2}, \mathrm{CuInS}_{2}$, and $\mathrm{CuGaSe}_{2}$ surfaces and interfaces give evidence for the formation of $\mathrm{Cu}$ vacancies when the Fermi level moves upwards in the band gap due to contact formation. The effect might be a key issue in understanding basic properties of solar cell devices based on these materials. (C) 1999 American Institute of Physics.
\end{abstract}

[S0003-6951(99)00516-1]

The Cu-chalcopyrite semiconductors $\mathrm{CuInSe}_{2}$, $\mathrm{CuGaSe}_{2}, \mathrm{CuInS}_{2}$, and their alloys (hereafter referred to as CIGS) have received considerable attention in recent years. They are analogs of the more common 2-6 compound semiconductors. Their physical properties, however, are more complex than those of their binary counterparts because of the metal d-state contribution to the valence band density of states. ${ }^{1-3}$ Research on ternary compounds is stimulated by their potential use in optoelectronic devices. CIGS compounds have been extensively explored as thin film solar cell absorber material. ${ }^{4,5}$ Within the last decade solar cells on the basis of thin film CIGS could be optimized such that efficiencies have exceeded 17\% (see, e.g., Ref. 6 and references therein).

Typically CIGS solar cells are made with $p$-type absorber material, $n$-type buffer layers (e.g., CdS), and a combination of intrinsic and highly conducting $\mathrm{ZnO}$ window layers. The basic parameters which determine the device performance are the heterojunction band discontinuities $\Delta E_{V}$ and $\Delta E_{C}$ of the different heterointerfaces and the doping densities of the layers. An idealized band diagram of a solar cell device is sketched in Fig. 1.

In Fig. 1 we assumed for simplicity a nondoped CdS buffer layer and neglected the possible influence of charged interface states. The given band discontinuities do not correspond to experimental values but are selected to give small barriers in the conduction band. It is generally accepted that the window and buffer layers do not contribute to the photoresponse because of their larger band gaps. The maximum photovoltage of the device is determined by the maximum band bending $e V_{b b}$, which is given by the distance between the Fermi level $E_{F}$ and the valence band maximum $E_{V}$ outside the space charge region and by the distance between $E_{F}$ and $E_{V}$ at the surface of the absorber. The latter is referred to as $\xi$ throughout this work. In Fig. $1 \xi$ represents the "highest" position of the Fermi level in the CIGS absorber. In general $\xi$ is determined from the overall charge neutrality condition and depends on the doping levels of the absorber and the $n^{+}-\mathrm{ZnO}$ window, as well as on the thicknesses of the $\mathrm{CdS}$ buffer and $i-\mathrm{ZnO}$ layers. Furthermore there is also a dependence of $\xi$ on the band discontinuities at the CIGS/CdS and the $\mathrm{CdS} / \mathrm{ZnO}$ interfaces, which is not considered here.

${ }^{a)}$ Electronic mail: aklein@hrzpub.tu-darmstadt.de
In principle, a larger band bending, and hence a larger photovoltage can be obtained when the band gap of the absorber is increased. Indeed an increase of open circuit photovoltage is observed in $\mathrm{Cu}(\mathrm{In}, \mathrm{Ga}) \mathrm{Se}_{2}$ solar cells with increasing Ga content. ${ }^{7,8}$ However, the photovoltage saturates for a certain Ga content which leads to a decrease in solar cell efficiency for larger band gap materials due to the reduced photocurrent. A possible explanation for this effect might be the change in band alignment at the CIGS/CdS interface. As the valence bands of $\mathrm{CuInSe}_{2}$ and $\mathrm{CuGaSe}_{2}$ are close in energy, ${ }^{3}$ similar valence band discontinuities $\Delta E_{V}$ can be expected for the $\mathrm{CdS} / \mathrm{CuInSe}{ }_{2}$ and the $\mathrm{CdS} / \mathrm{CuGaSe}_{2}$ interfaces. Increasing the $\mathrm{Ga}$ content will raise the conduction band above that of the CdS window as reported by Herberholz et al. ${ }^{8}$ The maximum band bending is then roughly given by the CdS conduction band position.

In this letter we will report on the formation of $\mathrm{Cu}$ vacancies, when $\xi$ is increased by band bending induced on clean $\mathrm{Cu}$-chalcopyrite surfaces. This intrinsic defect formation mechanism might also be responsible for the observed photovoltage saturation since it prevents the Fermi level from shifting closer to the conduction band via the introduction of acceptor states.

Our experimental results are summarized in Fig. 2. The relative contribution of $\mathrm{Cu} 3 d$ and $\mathrm{Se} 4 p$ orbitals to the valence band density of states is shown in Fig. 2(a). Its determination has been described in detail elsewhere. ${ }^{9}$

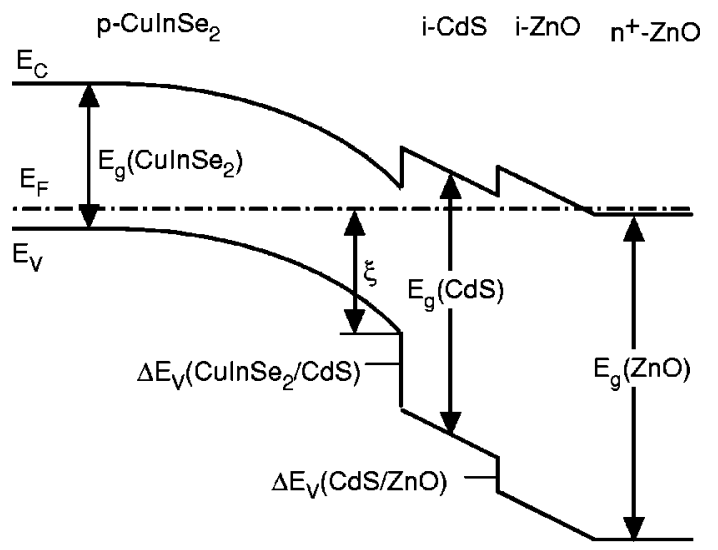

FIG. 1. Simplified band diagram of a solar cell device based on thin film $\mathrm{CuInSe}_{2}$ as light absorber. The quantity $\xi$ represents the maximum distance between the Fermi level and the valence band maximum in the absorber. 

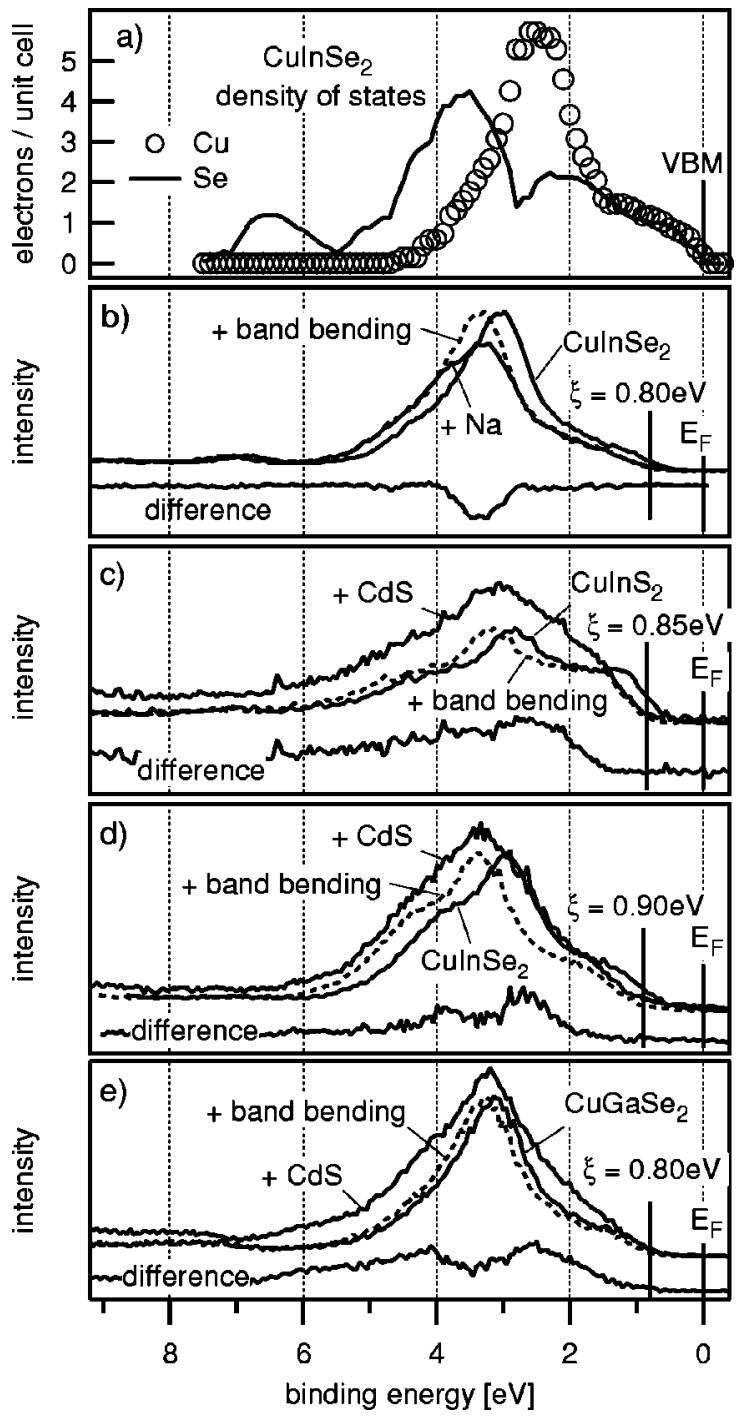

FIG. 2. (a) Partial density of states of CuInSe ${ }_{2}$ derived from energy dependent valence band photoelectron spectra (Ref. 9); (b)-(e) valence band spectra of clean and adsorbate covered CIGS surfaces excited with $h \nu=80 \mathrm{eV}$ synchrotron radiation (see Refs. 10-12). Binding energy shifts due to adsorbate induced band bending are shown by the dashed spectra. Fermi level positions $\xi$ after completed band bending are indicated. Difference spectra in (b), (d), and (e) show a characteristic intensity loss at $\mathrm{BE} \approx 3.4 \mathrm{eV}$.

Valence band photoelectron spectra, excited with $h \nu$ $=80 \mathrm{eV}$ synchrotron radiation, for different CIGS surfaces before and after deposition of $\mathrm{Na}$ or $\mathrm{CdS}$ are shown in Figs. 2(b) $-2(\mathrm{e})$. The clean surfaces exhibit Fermi level positions corresponding to $\xi=0.4-0.6 \mathrm{eV}$. For all examples shown in Fig. 2 the deposition of $\mathrm{Na}$ or $\mathrm{CdS}$ leads to an increase in binding energy due to downward band bending (for more details see Refs. 10-12). Values of $\xi$ after the band bending has been completed are indicated in the figure.

An increase in $\xi$ requires electron transfer from the adsorbate to the substrate which is readily understood for $\mathrm{Na}$ deposition because of its very small electronegativity. A more complete description of the interaction of $\mathrm{Na}$ with clean $\mathrm{CuInSe}_{2}$ surfaces has been presented recently. ${ }^{10}$ The band bending observed when CdS is deposited on CIGS surfaces $^{11,12}$ is generally explained by the intrinsic $n$-type doping of these films.

The difference spectra shown in Fig. 2(b) clearly indicates a loss in intensity at a binding energy $(\mathrm{BE}) \approx 3.4 \mathrm{eV}$, corresponding to $\mathrm{BE} \approx 2.6 \mathrm{eV}$ with respect to the valence band maximum (VBM). At this binding energy mainly the $\mathrm{Cu} 3 d$ levels contribute to the emission intensity as shown in Fig. 2(a). Any contribution of $\mathrm{Na}$ to the valence band spectra can be neglected because of the small photoionization cross section of the Na $3 s$ levels at this excitation energy. ${ }^{13}$ Therefore it has been argued that the intensity loss is due to removal of $\mathrm{Cu}$ from the surface by formation of $\mathrm{Cu}$ vacancies and subsequent diffusion of $\mathrm{Cu}$ into the bulk. ${ }^{10}$

During interface formation between $\mathrm{CdS}$ and $\mathrm{CuInS}_{2}$ the valence bands can be described by a superposition of $\mathrm{CuInS}_{2}$ and $\mathrm{CdS}$ valence band emissions. ${ }^{12}$ Hence the difference spectra between a surface partially covered with $\mathrm{CdS}$ and the bare $\mathrm{CuInS}_{2}$ surface [Fig. 2(c)] resembles that of the thick $\mathrm{CdS}$ film. The situation is different for partially CdS covered $\mathrm{CuInSe} \mathrm{C}_{2}$ and $\mathrm{CuGaSe}_{2}$ surfaces, where again an intensity loss at $\mathrm{BE} \approx 2.6 \mathrm{eV}$ below the VBM is observed in the difference spectra as evident from Figs. 2(d) and 2(e). The effect is less pronounced for $\mathrm{CuInSe}_{2}$. In terms of the interpretation given for $\mathrm{Na} / \mathrm{CuInSe} e_{2}$ a loss of $\mathrm{Cu}$ from the surface also occurs during CdS deposition on $\mathrm{CuInSe}_{2}$ and $\mathrm{CuGaSe}_{2}$, while no effect is observed for $\mathrm{CuInS}_{2}$.

Similar to the case of $\mathrm{Na}$ deposition, which leads to a chemical decomposition of the surface only for higher coverages, ${ }^{10}$ a chemical reaction is not considered as the origin for the observed $\mathrm{Cu}$ reduction in the case of $\mathrm{CdS}$ deposition. In contrast, we suggest that a fundamental mechanism, namely the movement of the Fermi level towards the conduction band, is responsible for the formation of the $\mathrm{Cu}$ vacancies.

Fermi level dependent defect formation is a long known mechanism in compound semiconductors and explains a number of experimental observations (see, e.g., Refs. 14 and 15 and references therein). One related example is the maximum doping density in these materials. The doping limits occur because shifting the Fermi level above (for $n$-type doping) or below (for $p$-type doping) some critical value by intentionally introduced additional dopants, thermodynamically leads to the formation of lattice defects, compensating the higher dopant concentration.

Zhang et al. have presented detailed calculations of the defect properties of CuInSe ${ }_{2}{ }^{15,16}$ Their results show that the energy needed to create a $\mathrm{Cu}$ vacancy decreases with increasing Fermi level position and might even become negative. However, a detailed explanation of the proposed Fermi level dependent defect formation needs to correlate the calculated energy values of the defect system (e.g., $V_{\mathrm{Cu}}+\mathrm{Cu}_{i}$ ) including pair formation energies with experimental determinations of the involved changes.

As deduced from Fig. 2 removal of $\mathrm{Cu}$ from $\mathrm{CuInSe}_{2}$ and $\mathrm{CuGaSe}_{2}$ surfaces is observed when $\xi$ increases above $\approx 0.80 \mathrm{eV}$. This value agrees very well with observed Fermi level positions at surfaces of $\mathrm{Cu}$-poor $\mathrm{Cu}(\mathrm{In}, \mathrm{Ga}) \mathrm{Se}_{2}$ films. ${ }^{17}$ While $\mathrm{Cu}$-rich surfaces are generally $p$ type, the surface converts to $n$ type when the films are grown with $\mathrm{Cu}$-poor stoichiometry. However, throughout the whole In-Ga alloy range $\xi$ is constant at $\xi \approx 0.85 \mathrm{eV}$ for $\mathrm{Cu}$ poor films. ${ }^{17}$ This agrees well with the statement of Zhang et al. that the maximum doping level is constant in these compounds on an absolute energy scale, ${ }^{15}$ together with the comparable ener- 
gies of the $\mathrm{CuInSe}_{2}$ and $\mathrm{CuGaSe}_{2}$ valence band edges. ${ }^{3,15}$

Assuming the energy level, where $\mathrm{Cu}$ vacancies are formed, to be identical in CIGS compounds, the lack of $\mathrm{Cu}$ vacancy formation in $\mathrm{CuInS}_{2}$ at comparable values of $\xi$ [Fig. $2(\mathrm{c})]$ can be attributed to its different valence band position. According to the literature ${ }^{3,15}$ the valence band maximum of $\mathrm{CuInS}_{2}$ has a $0.2-0.3 \mathrm{eV}$ higher binding energy compared to those of $\mathrm{CuInSe}_{2}$ and $\mathrm{CuGaSe}_{2}$. This theoretical result is supported by the different valence band discontinuities between $\mathrm{CdS}$ and these compounds $\left[\Delta E_{V}\left(\mathrm{CdS} / \mathrm{CuInS}_{2}\right)\right.$ $\left.=0.6 \mathrm{eV} ;{ }^{12} \Delta E_{V}\left(\mathrm{CdS} / \mathrm{CuInSe}_{2}\right)=0.8 \mathrm{eV}^{11}\right)$.

Consequently, larger values of $\xi$, and hence larger photovoltages, should be possible with $\mathrm{CuInS}_{2}$. However, the conversion efficiencies of the $\mathrm{Cu}(\mathrm{In}, \mathrm{Ga}) \mathrm{Se}_{2}$ solar cells are still superior to those obtained with sulfide films. ${ }^{6}$ One reason might be that the interface to the CdS buffer layer, which could introduce significant carrier losses, becomes more important in the sulfides. ${ }^{18}$ This interface is of reduced importance for the selenides which show a type inversion at the surface related to the commonly known ordered vacancy compounds (OVCs), moving the space charge region away from the CdS/CIGS interface. ${ }^{19}$ In addition the possibility of $\mathrm{CuInSe}_{2}$ to form OVCs also allows for larger $\xi$ because of the different band edge energies. ${ }^{15}$

We have presented photoelectron spectroscopy results of several interfaces formed with $\mathrm{CuInSe}_{2}$ and related compounds. The interface formation is accompanied by downward band bending and leads to a characteristic intensity loss in the valence bands which is explained by an intrinsic defect formation mechanism. The removal of $\mathrm{Cu}$ from the surface via (i) $\mathrm{Cu}$ vacancy formation as a result of the movement of the Fermi level towards the conduction band, and (ii) diffusion of $\mathrm{Cu}$ into the bulk, requires, of course, more detailed experimental verification with a better control of surface potential. However, this fundamental process seems to be of particular importance with CIGS solar cells and might present a key in understanding several issues of device performance.

${ }^{1}$ J. L. Shay and J. H. Wernick, Ternary Chalcopyrite Semiconductors (Pergamon, Oxford, 1975).

${ }^{2}$ J. E. Jaffe and A. Zunger, Phys. Rev. B 29, 1882 (1984).

${ }^{3}$ S.-H. Wei and A. Zunger, J. Appl. Phys. 78, 3846 (1995).

${ }^{4}$ A. Rockett and R. W. Birkmire, J. Appl. Phys. 70, R81 (1991).

${ }^{5}$ V. Nadenau, D. Braunger, D. Hariskos, M. Kaiser, C. Köble, A. Oberacker, M. Ruckh, U. Rühle, R. Schäffler, D. Schmid, T. Walter, S. Zweigart, and H. W. Schock, Prog. Photovoltaics 3, 363 (1995).

${ }^{6} \mathrm{~K}$. Bücher and S. Kunzelmann, Proceedings of the 14th European Photovoltaic Solar Energy Conference (H. S. Stephens, Bedford, 1997), p. 272.

${ }^{7}$ W. N. Shafarman, R. Klenk, and B. E. McCandless, J. Appl. Phys. 79, 7324 (1996)

${ }^{8}$ R. Herberholz, V. Nadenau, U. Rühle, C. Köble, H. W. Schock, and B. Dimmler, Sol. Energy Mater. Sol. Cells 49, 227 (1997).

${ }^{9}$ T. Löher, A. Klein, C. Pettenkofer, and W. Jaegermann, J. Appl. Phys. 81, 7806 (1997).

${ }^{10}$ A. Klein, T. Löher, C. Pettenkofer, and W. Jaegermann, J. Appl. Phys. 80, 5039 (1996)

${ }^{11}$ T. Löher, W. Jaegermann, and C. Pettenkofer, J. Appl. Phys. 77, 731 (1995).

${ }^{12}$ A. Klein, T. Löher, C. Pettenkofer, and W. Jaegermann, Appl. Phys. Lett. 70, 1299 (1997)

${ }^{13}$ J. Yeh and I. Lindau, At. Data Nucl. Data Tables 32, 1 (1985).

${ }^{14}$ W. Walukiewicz, J. Vac. Sci. Technol. B 6, 1257 (1988).

${ }^{15}$ S. B. Zhang, S.-H. Wei, and A. Zunger, J. Appl. Phys. 83, 3192 (1998).

${ }^{16}$ S. B. Zhang, S.-H. Wei, and A. Zunger, Phys. Rev. Lett. 78, 4059 (1997); S. B. Zhang, S.-H. Wei, A. Zunger, and H. Katayama-Yoshida, Phys. Rev. B 57, 9642 (1998).

${ }^{17}$ D. Schmid, M. Ruckh, and H. W. Schock, Appl. Surf. Sci. 103, 409 (1996).

${ }^{18}$ R. Scheer, M. Wilhelm, H. J. Lewerenz, H. W. Schock, and L. Stolt, Sol. Energy Mater. Sol. Cells 49, 299 (1997).

${ }^{19}$ D. Schmid, M. Ruckh, F. Grunwald, and H. W. Schock, J. Appl. Phys. 73, 2902 (1993).

This article may be downloaded for personal use only. Any other use requires prior permission of the author and AIP Publishing. This article appeared in Appl. Phys. Lett. 74, 2283 (1999) and may be found at https://doi.org/10.1063/1.123825.

Available under only the rights of use according to UrhG. 